\title{
Quorum-sensing signals in the microbial community of the cabbage white butterfly larval midgut
}

\author{
Bradley R Borlee ${ }^{1,5}$, Grant D Geske ${ }^{2}$, Courtney J Robinson ${ }^{3,4}$, Helen E Blackwell ${ }^{2}$ \\ and Jo Handelsman ${ }^{1,3}$ \\ ${ }^{1}$ Department of Plant Pathology, University of Wisconsin-Madison, Madison, WI, USA; ${ }^{2}$ Department of \\ Chemistry, University of Wisconsin-Madison, Madison, WI, USA ${ }^{3}$ Department of Bacteriology, \\ University of Wisconsin-Madison, Madison, WI, USA and ${ }^{4}$ Microbiology Doctoral Training Program, \\ University of Wisconsin-Madison, Madison, WI, USA
}

\begin{abstract}
The overall goal of this study was to examine the role of quorum-sensing (QS) signals in a multispecies microbial community. Toward this aim, we studied QS signals produced by an indigenous member and an invading pathogen of the microbial community of the cabbage white butterfly (CWB) larval midgut (Pieris rapae). As an initial step, we characterized the QS system in Pantoea CWB304, which was isolated from the larval midgut. A luxl homolog, designated panl, is necessary for the production of $\mathrm{N}$-acyl-L-homoserine lactones (AHLs) by Pantoea CWB304. To determine whether AHL signals are exchanged in the alkaline environment of the midgut, we constructed AHL-sensing bioluminescent reporter strains in Pantoea CWB304 and a pan/ mutant of this strain. In the gut of the CWB larvae, the reporter in an AHL-deficient Pantoea CWB304 detected AHLs when coinoculated with the wild type. To study the role of AHL signals produced by a community invader, we examined pathogenesis of Pseudomonas aeruginosa PAO1 in CWB larvae. Mortality induced by $P$. aeruginosa PAO1 was significantly reduced when signaling was interrupted by either a potent chemical inhibitor of QS or mutations in the lasl and rhll AHL synthases of $P$. aeruginosa PAO1. These results show that AHLs are exchanged among bacteria in the alkaline gut of CWB larvae and contribute to disease caused by $P$. aeruginosa PAO1.
\end{abstract}

The ISME Journal (2008) 2, 1101-1111; doi:10.1038/ismej.2008.70; published online 24 July 2008

Subject Category: microbe-microbe and microbe-host interactions

Keywords: quorum sensing; $\mathrm{N}$-acyl-L-homoserine lactones; cell-cell communication; multispecies community; Pseudomonas aeruginosa PAO1; cabbage white butterfly

\section{Introduction}

Quorum sensing (QS) is the cell density-dependent regulation of gene expression that enables members of a bacterial population to work together to accomplish tasks that would be impossible or useless for a single cell. Genes regulated by QS dictate a diversity of physiological traits in various bacteria that are often involved in the establishment of mutualistic symbioses (Lithgow et al., 2000; Visick et al., 2000) and pathogenic relationships (Cui et al., 1995; Pearson et al., 2000). In

Correspondence: J Handelsman, Department of Bacteriology, University of Wisconsin-Madison, 6159 Microbial Sciences, 1550 Linden Drive, Madison, WI 53706, USA.

E-mail: joh@bact.wisc.edu.

${ }^{5}$ Current address: Department of Microbiology, University of Washington School of Medicine, 1959 NE Pacific St, Room K-360, Seattle, WA 98195-7242, USA.

Received 24 April 2008; revised 22 June 2008; accepted 23 June 2008; published online 24 July 2008
Proteobacteria, communication is mediated by $N$-acyl-L-homoserine lactone (AHL) signal molecules (Manefield and Turner, 2002) and homologs of LuxI and LuxR proteins (Fuqua et al., 1994). AHLs are synthesized by LuxI-type synthases and interact with LuxR-type regulator proteins to regulate the expression of specific genes positively or negatively (Engebrecht and Silverman, 1984; von Bodman et al., 1998). Although bacterial QS systems differ in the chemical structures of the signals, signal relay mechanisms and target genes they regulate, many bacteria share the ability to communicate with each other to coordinate gene expression and ultimately their behavior in the community (Miller and Bassler, 2001).

Despite advances in understanding the role of signaling in bacteria-host interactions, the role of signaling in multispecies bacterial communities remains largely unexplored. Some studies suggest that interspecies communication, signal mimicry and signal degradation play a role in interspecies 
interactions. Many bacteria produce AHLs that other species can recognize. In the rhizosphere, Pseudomonas putida responds to AHLs produced by indigenous bacteria (Steidle et al., 2001), in mixed biofilms, Burkholderia cepacia responds to AHLs produced by Pseudomonas aeruginosa (Riedel et al., 2001) and Escherichia coli and Salmonella enterica serovar Typhimurium both detect AHLs produced by other species with the SdiA receptor, a predicted LuxR homolog (Michael et al., 2001). Molecules that functionally resemble natural ligands also modulate signaling networks by signal mimicry. Natural and synthetic furanones that mimic AHLs regulate AHLmediated QS systems in a positive and negative manner (Givskov et al., 1996; Martinelli et al., 2004). Signal mimics from uncultured bacteria associated with the midguts of gypsy moth larvae that induce QS have also been identified by a metagenomic analysis (Guan et al., 2007). Signal degradation may also influence signaling in multispecies communities (Leadbetter and Greenberg, 2000; Dong et al., 2002; Carlier et al., 2003; Lin et al., 2003; Park et al., $2003,2005)$. These examples illustrate some of the mechanisms by which microorganisms respond to signal mimics and possibly modulate QS networks in multispecies communities. Given the importance of QS in coordination and regulation of gene expression in single species, it seems likely that it plays a prominent role in the context of the entire community.

To study signaling in a multispecies community, we chose the midgut of the cabbage white butterfly (CWB) larva because it harbors a community of only a few bacterial species. This environment is intriguing because AHLs are typically unstable in alkaline $\mathrm{pH}$ environments, and the average $\mathrm{pH}$ of the midgut of larvae fed sterile artificial diet has been measured to be $8.68 \pm 0.53$ (unpublished); other studies have reported the $\mathrm{pH}$ to be 7.3-7.6 (Berenbaum, 1980; Wittstock et al., 2004). The gut community can be manipulated by amending the insect's sterile artificial diet with compounds or bacterial strains. When the larvae are reared on sterile artificial diet, the community is estimated to contain an average of 14 species of bacteria, of which the majority are uncultivated Proteobacteria. A more detailed report of the CWB midgut community composition will be published elsewhere (CJ Robinson, unpublished data). We reasoned that AHL-mediated intraspecies and interspecies communication between these bacteria could be pervasive.

We also sought to investigate the role of AHL signaling by invasive pathogens in this community. CWB larvae are susceptible to the opportunistic pathogen $P$. aeruginosa, which uses AHL-mediated QS to control virulence gene expression during infection of diverse eukaryotic hosts including plants, nematodes, insects and mammals under the control of AHL-mediated QS (Pearson et al., 2000; Lesprit et al., 2003). P. aeruginosa produces distinct AHLs, N-butyryl homoserine lactone (Pearson et al., 1995) and $\mathrm{N}$-(3-oxododecanoyl)-L-homoserine lactone (Pearson et al., 1994), which interact with the LasR, RhlR and QscR transcriptional regulators to direct the transcription of distinct but overlapping regulons that include virulence genes (Latifi et al., 1995; Pearson et al., 1997; Lequette et al., 2006).

In this study, we report that AHL-mediated QS is active in the microbial community of the CWB larval gut. Furthermore, we demonstrate that QS signals play a role in pathogenesis by $P$. aeruginosa, which can be attenuated by administering a non-native AHL antagonist. The study establishes the CWB larval midgut as a compelling model system for study of microbial community structure and function.

\section{Materials and methods}

Bacterial strains and growth conditions

The bacterial strains and plasmids used in this study are listed in Table 1. All strains were grown in Luria-Bertani (LB) medium at $28^{\circ} \mathrm{C}$. Antibiotics were added as required at final concentrations of $20 \mathrm{mg}$ tetracycline per $1,50 \mathrm{mg}$ trimethoprim per 1 and $25 \mathrm{mg}$ nalidixic acid per $\mathrm{l}$. Bacteria that were fed to CWB larvae were prepared from stationary phase cultures by centrifugation, washed twice and resuspended in $1 / 10$ of the original volume in LB medium. Ten microliters of the washed and concentrated bacterial suspension was applied to each disk of insect diet.

Isolation and identification of AHL-producing bacteria Bacterial strains were isolated from the midgut of CWB larvae and identified by $16 \mathrm{~S}$ rRNA gene sequencing as described previously (Broderick et al., 2004). Isolates were screened for AHL production on LB agar plates in a cross-streaking assay against the AHL reporter strains Chromobacterium violaceum CV026 (McClean et al., 1997), E. coli pJBA132 (Andersen et al., 2001) and P. putida F117 pKRC12 (Steidle et al., 2001) as described previously (Williamson et al., 2005).

\section{Random insertion mutagenesis of Pantoea CWB304}

Random transposon mutations were generated in Pantoea CWB304 with the EZ-Tn5〈DHFR-1〉Tnp Transposome kit (Epicentre, Madison, WI, USA) according to the manufacturer's instructions. Transposase complexes were introduced by electroporation into Pantoea CWB304 electrocompetent cells as directed in the operating manual for the Gene Pulser transfection apparatus for E. coli (Bio-Rad Laboratories, Hercules, CA, USA), except that cultures were incubated at $28^{\circ} \mathrm{C}$ for preparation of electrocompetent cells. Electroporated cells were allowed to recover for $2 \mathrm{~h}$ at $28^{\circ} \mathrm{C}$ with shaking, plated on $\mathrm{LB}$ agar containing trimethoprim and incubated at $28^{\circ} \mathrm{C}$ to select for transposon insertion. 
Table 1 Bacterial strains and plasmids

\begin{tabular}{|c|c|c|}
\hline Strain or plasmid & Relevant characteristics & Source or reference \\
\hline Pantoea CWB301 & $\begin{array}{l}\text { Bacterial isolate from cabbage white butterfly } \\
\text { larvae, AHL producer }\end{array}$ & This study \\
\hline Pantoea CWB304 & $\begin{array}{l}\text { Bacterial isolate from cabbage white butterfly } \\
\text { larvae, AHL producer, Nal }{ }^{\mathrm{R}} \text { CWB301 } \\
\text { derivative }\end{array}$ & This study \\
\hline Pantoea CWB304 panI::Tn5 & $\begin{array}{l}\text { Tn5 mutant, } \operatorname{Trm}^{\mathrm{R}}, \mathrm{Nal}^{\mathrm{R}} \text {, AHL-deficient } \\
\text { CWB304 derivative }\end{array}$ & This study \\
\hline Pseudomonas aeruginosa PAO1 & Wild-type strain, AHL producer & Pearson et al. (1997) \\
\hline $\begin{array}{l}\text { Pseudomonas aeruginosa PAO1- } \\
\text { JP2 }\end{array}$ & $\begin{array}{l}\Delta r h l I:: T n 501-2, \Delta l a s I:: \text { Tet, derivative of PAO1, } \\
\mathrm{Hg}^{\mathrm{R}} \text { and } \mathrm{Tet}^{\mathrm{R}} \text {, AHL-deficient }\end{array}$ & Pearson et al. (1997) \\
\hline $\begin{array}{l}\text { Chromobacterium violaceum } \\
\text { CV026 }\end{array}$ & AHL-deficient, AHL reporter strain & McClean et al. (1997) \\
\hline Escherichia coli DH5 $\alpha$ & recA1 and endA1 cloning strain & $\begin{array}{l}\text { Invitrogen, Carlsbad, } \\
\text { CA, USA }\end{array}$ \\
\hline $\begin{array}{l}\text { Agrobacterium tumefaciens } \\
\text { NTL4 pZLR4 }\end{array}$ & AHL-deficient, AHL reporter strain, $\mathrm{Gm}^{\mathrm{R}}$ & Piper et al. (1993) \\
\hline $\begin{array}{l}\text { Escherichia coli MT102 } \\
\text { pJBA132 }\end{array}$ & AHL-deficient, AHL reporter strain, Tet $^{\mathrm{R}}$ & Andersen et al. (200 \\
\hline $\begin{array}{l}\text { Pseudomonas putida F117 } \\
\text { pKRC12 }\end{array}$ & $\begin{array}{l}\text { AHL-deficient derivative of } P \text {. putida IsoF, } \\
\Delta p p u I, \text { AHL reporter strain } \\
\text { pKR-C12: pBBR1MCS-5 carrying } \\
\mathrm{P}_{\text {las }}-\text { gfp(ASV)-P } \text { Iac }_{\text {lasR; }} \text {; based on components } \\
\text { of the } P \text {. aeruginosa las } \mathrm{QS} \text { system; } \mathrm{Gm}^{\mathrm{R}}\end{array}$ & Steidle et al. (2001) \\
\hline pSB401 & $\begin{array}{l}\mathrm{P}_{l u x I}-l u x C D A B E \text { transcriptional fusion, } \operatorname{lu} x R^{+} \text {, } \\
\text { Tet }^{\mathrm{R}}\end{array}$ & Winson et al. (1998) \\
\hline pPanlux & $\mathrm{P}_{\text {panI }}-l u x C D A B E$ transcriptional fusion, $\mathrm{Tet}^{\mathrm{R}}$ & This study \\
\hline pGEM-5Zf(+) & Cloning vector, $\mathrm{Amp}^{\mathrm{R}}$ & Promega \\
\hline pGEM-T Easy & TA cloning vector, $A_{m p}{ }^{R}$ & Promega \\
\hline
\end{tabular}

Abbreviations: AHL, $N$-acyl-L-homoserine lactone; $\mathrm{Gm}^{\mathrm{R}}$, gentamicin resistance; $\mathrm{Hg}^{\mathrm{R}}$, mercuric chloride resistance; $\mathrm{Nal}^{\mathrm{R}}$, nalidixic acid resistance; $\mathrm{Tet}^{\mathrm{R}}$, tetracycline resistance; $\operatorname{Trm}^{\mathrm{R}}$, trimethoprim resistance.

\section{Identification and characterization of AHL-deficient Pantoea mutants}

Trimethoprim-resistant Pantoea mutants were patched pairwise with the AHL reporter strain C. violaceum CV026. Presence of AHL is indicated by the production of violacein. Pantoea mutants that did not induce violacein production in C. violaceum CV026 were further analyzed by sequence analysis of the genomic DNA flanking the transposon. Genomic DNA from the Pantoea mutants was isolated with the Easy-DNA genomic DNA extraction kit (Invitrogen, Carlsbad, CA, USA). Genomic DNA was digested with SacII (Promega, Madison, WI, USA) and ligated into pGEM-5Zf $(+)$ (Promega), which was linearized with SacII. The ligation products were electroporated into E. coli $\mathrm{DH} 5 \alpha$ that was plated on LB agar containing trimethoprim and incubated at $37^{\circ} \mathrm{C}$ to select clones containing the transposon with flanking DNA. Plasmid DNA was isolated from trimethoprim-resistant transformants and sequenced with primers complementary to the transposon. Primer walking with custom-designed primers obtained additional nucleotide sequences. BigDye Terminator (v. 3.1; Applied Biosystems, Foster City, CA, USA) sequencing was conducted at the University of Wisconsin-Madison DNA-sequencing facility. Sequence reads were assembled with Seqman (DNASTAR, Madison, WI, USA).
Characterization of AHLs produced by Pantoea strain CWB304

Culture extracts for thin layer chromatography (TLC) analysis were prepared from $50 \mathrm{ml}$ cultures grown to stationary phase at $28{ }^{\circ} \mathrm{C}$. Bacteria were removed by centrifugation, and the supernatants were extracted twice with $50 \mathrm{ml}$ of acidified ethyl acetate $(0.1 \mathrm{ml}$ glacial acetic acid per l). Residues were dried, then dissolved in a final volume of $50 \mu \mathrm{l}$ acidified ethyl acetate and stored at $-20^{\circ} \mathrm{C}$. One-microliter samples of extracted culture supernatants and synthetic AHL standards were applied to $\mathrm{C}_{18}$ reversed-phase TLC plates (200-m layer; JT Baker, San Francisco, CA, USA) and developed with methanol/water (60:40, $\mathrm{vol} / \mathrm{vol}$ ). Solvents were evaporated, and the plates were analyzed for the presence of AHLs with an Agrobacterium tumefaciens AHL indicator strain NTL4 pZLR4 (Piper et al., 1993). The AHL indicator was grown to stationary phase and $5 \mathrm{ml}$ of culture was added to $95 \mathrm{ml}$ of $\mathrm{LB}$ medium maintained at $50{ }^{\circ} \mathrm{C}$ amended with $7.5 \mathrm{~g}$ agar per $\mathrm{l}$ and $50 \mu \mathrm{g} \mathrm{ml}^{-1} 5$ bromo-4-chloro-3-indolyl $\beta$-D-galactopyranoside (X$\mathrm{Gal}$ ). The agar suspension containing the bacteria was mixed briefly with a vortex mixer and $50 \mathrm{ml}$ was applied to the TLC plate by spraying $(250-\mathrm{ml}$ sprayer; Kontes, Vineland, NJ, USA). After the agar solidified, TLC plates were incubated at $28{ }^{\circ} \mathrm{C}$ for $18 \mathrm{~h}$ in Big Blue Trays (Stratagene, La Jolla, CA, USA). $R_{\mathrm{f}}$ values for samples were compared with 
AHL standards, which were the gifts from Dr Ronald Binder (USDA ARS Pacific West Area).

\section{Construction and characterization of Pantoea QS reporters}

The luxCDABE-based AHL sensor plasmid, pSB401, was introduced by electroporation into the wildtype Pantoea strain and the Pantoea AHL-negative mutant to monitor QS activity. To monitor the regulation of panI, we constructed a panI transcriptional fusion with the bacterial luciferase reporter luxCDABE and introduced this construct into Pantoea CWB304. The promoter region and partial coding region of panI was PCR-amplified using the primers pantprofor $\left(5^{\prime}\right.$-AACAGCCCAGTCAAGTC GATCCTT- $3^{\prime}$ ) and pantprorev (5'-TTGACGGGAC TTGTAGTTGCCCTT-3'). The 397-bp amplicon was TA-cloned into pGEM-T Easy (Promega) as suggested by the manufacturer to create plasmid pGTEpantpro. The reporter plasmid pSB401 was linearized by EcoRI digestion and purified by gel electrophoresis and gel extraction to remove the 1$\mathrm{kb}$ region that encodes luxR and the luxI promoter, resulting in a linearized vector with a promoterless luxCDBAE operon. Plasmid pGTEpantpro was linearized by EcoRI digestion, and the panI promoter and partial coding region were purified by gel electrophoresis and gel extraction and ligated into the modified pSB401 promoterless vector to produce plasmid pPanlux, which was introduced by electroporation into Pantoea CWB304 panI::Tn5.

All of the reporter strains were monitored for bioluminescence activity in the presence and absence of synthetic $50 \mathrm{nM} \mathrm{N}$-(3-oxo-hexanoyl)-Lhomoserine lactone (3-oxo-C6 AHL). Bacterial cells from overnight cultures were concentrated, washed twice and inoculated into $\mathrm{LB}$ medium at an $\mathrm{OD}_{600}$ of 0.04. Cells were dispensed into 96-well Costar 3603 plates (Corning Inc., Corning, NY, USA) to a final volume of $100 \mu \mathrm{l}$. The cultures were incubated at $28{ }^{\circ} \mathrm{C}$ with constant agitation.

Bioluminescence and absorbance $\left(\mathrm{OD}_{600}\right)$ were measured with a Wallac Victor2 plate reader (PerkinElmer Life and Analytical Sciences, Wellesley, MA, USA), and specific luminescence was defined as relative luminescence per unit of absorbance $\left(\mathrm{OD}_{600}\right)$. Bioluminescence of Pantoea CWB304 pPanlux was also monitored during growth in LB medium, and the bioluminescence of Pantoea CWB304 panI::Tn5 pPanlux was measured in response to exogenous provision of $\mathrm{N}$-hexanoyl-L-homoserine lactone (C6 AHL), $N$-octanoyl-L-homoserine lactone (C8 AHL), 3-oxo-C6 AHL and N-(3-oxo-octanoyl)-L-homoserine lactone (3-oxo-C8 AHL) at final concentrations of 5, $10,50,250$ and $500 \mathrm{nM}$.

\section{CWB larvae rearing}

CWB eggs (Carolina Biological Supply Company, Burlington, NC, USA) were surface-sterilized by soaking in a solution of $1 \%$ Tween 80 and $2 \%$ bleach for $3 \mathrm{~min}$, rinsed twice with sterile distilled water and dried under a sterile air stream in a laminar flow hood. Sterile artificial diet (USDA, Hamden formula) was added to the petri plates containing the eggs, and fresh sterile artificial diet was supplied every $72 \mathrm{~h}$ or as it was consumed. Plates were partially sealed with parafilm and incubated in an environmentally controlled chamber maintained at $25^{\circ} \mathrm{C}$ with a photoperiod of 16-8 h (light-dark).

Visualization of reporter gene activity in insecta CWB larvae were reared to middle of the fourth instar growth stage and starved for $4 \mathrm{~h}$. Thereafter, individual larvae were transferred to wells of 12well Costar cell culture plates (Corning Inc.), which contained artificial diet disks to which various bacterial strains were added. Larvae were fed on the diet disks for $18 \mathrm{~h}$. After feeding, larvae that had consumed the diet were starved for $6 \mathrm{~h}$ before imaging. Bioluminescence emitted from live larvae and intact guts dissected from larvae were measured with an IVIS BLI 100 CCD camera (Xenogen, Alameda, CA, USA). Dissection of intact guts was performed as previously described (Broderick et al., 2004).

\section{Plasmid transfer in insecta}

To determine whether strains of Pantoea transferred plasmids in the guts of CWB larvae, Pantoea CWB304 panI::Tn5 was mixed in a 1:1 ratio with Pantoea CWB304 pSB401 and fed to CWB larvae as described. After feeding, bacteria isolated from dissected guts were plated on selective media containing trimethoprim and tetracycline to determine whether plasmid transfer had occurred.

\section{Growth of P. aeruginosa PAO1 in CWB larvae}

To characterize the growth of $P$. aeruginosa PAO1 in CWB larvae, we monitored the population of $P$. aeruginosa PAO1 as a function of time. The guts from CWB larvae fed on unamended diet and diet amended with $P$. aeruginosa PAO1 were harvested at time intervals of 1,3 and 5 days after feeding. Dissection of guts and quantification of bacteria in the guts were performed as previously described (Broderick et al., 2004). To determine whether $P$. aeruginosa PAO1 survived on sterile artificial diet, bacterial suspensions were applied to diet disks as described above.

\section{P. aeruginosa pathogenicity assays}

Diet disks were amended with various compounds and bacterial strains. The indole QS inhibitor compound, $N$-(indole-3-butanoyl)-L-homoserine lactone was dissolved in ethyl acetate at a concentration of $15 \mathrm{mg} \mathrm{ml}^{-1}$. $\mathrm{N}$-(indole-3-butanoyl)-L-homoserine lactone (referred to as 'indole analog' 
throughout) was synthesized and purified according to methods described previously (purity $\geqslant 95 \%$ )

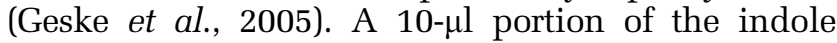
compound solution or ethyl acetate (control) was added to each diet disk in two aliquots of $5 \mu$ l. Ethyl acetate was evaporated from the samples in a sterile laminar flow hood. A 10- $\mu$ l aliquot of washed and concentrated bacterial suspension or LB medium (control) was applied to each disk. Larvae reared to the fourth instar were transferred to petri dishes that contained artificial diet disks with treatments after $4 \mathrm{~h}$ of withholding food. Each feeding treatment was replicated three times. Mortality of the larvae was assessed every $24 \mathrm{~h}$ until the larvae initiated pupation.

\section{Nucleotide sequence accession numbers}

The nucleotide sequence of panI and panR have been submitted to GenBank databases under accession numbers EU780668 and EU780669, respectively. The 16S rRNA gene sequence of Pantoea CWB304 has been submitted to GenBank databases under accession number EU780667.

\section{Results}

A larval gut inhabitant produces AHLs

As a first step in determining whether QS signals are important in the alkaline environment of the lepidopteran midgut, we surveyed the cultivated members of the CWB larval gut community for the ability to stimulate AHL biosensor strains by crossstreaking. We identified a bacterial isolate, CWB304, that induces violacein production in the $C$. violaceum CV026 AHL sensor and green fluorescent protein expression in the E. coli JB525 AHL sensor, both of which are responsive to short-chain AHLs, but did not stimulate the AHL sensor strain, P. putida F117 pKRC12, which responds to longer chain AHLs. The 16S rRNA gene sequence from CWB304 is 97\% identical to Pantoea sp. PPE7. Thus, we have designated this strain Pantoea CWB304. Further phylogenetic analyses of the isolate will be presented elsewhere.

Ethyl acetate extracts of cell-free supernatant from Pantoea CWB304 cultures retained the ability to induce AHL reporter strains. Organic extracts applied to TLC and visualized with the application of the A. tumefaciens NTL4 pZLR4 AHL sensor revealed the presence of four different AHLs in multiple extractions and chromatographs (Figure 1). On the basis of the mobility of the AHLs detected and comparison to synthetic standards (Figure 1), we tentatively identified these molecules as $\mathrm{C} 7$ AHL, C8 AHL, 3-oxo-C6 AHL and 3-oxo-C8 AHL.

Characterization of a gene responsible for AHL production in Pantoea CWB304

Random transposon mutants of Pantoea CWB304 were screened for loss of production of AHL by testing them for the ability to induce violacein production in $C$. violaceum strain CV026 (Figure 2). Out of the 11228 mutants screened, three failed to activate the CV026 AHL sensor strain. All three of the mutants contained transposon insertions in a homolog of the luxI synthase, which is divergently transcribed from a flanking luxR homolog. We designated the genes panI and panR based on the phenotype of the panI mutant and sequence similarity to luxI and luxR homologs, respectively. BlastX (Altschul et al., 1997) results indicate that the deduced amino-acid sequence of PanI is $59 \%$ identical and $74 \%$ similar to HslI from Pectobacterium carotovorum, and PanR is $45 \%$ identical and $69 \%$ similar to SmaR from Serratia sp. ATCC 39006. We failed to detect AHLs in cultures of the panI mutant after extraction with acidified ethyl acetate and TLC analysis (Figure 1).

\section{Bioluminescent AHL biosensors in Pantoea CWB304}

We constructed several reporter strains in which bioluminescence is a measure of AHL-mediated gene regulation to detect signal exchange in guts of CWB larvae. We introduced pSB401 into the wildtype Pantoea and the AHL-deficient mutant. The AHL reporter plasmid pSB401 is a transcriptional fusion of luxI promoter region and the bacterial luciferase reporter luxCDABE under transcriptional control of LuxR (Winson et al., 1998), which is activated by short-chain AHLs. Bioluminescence is

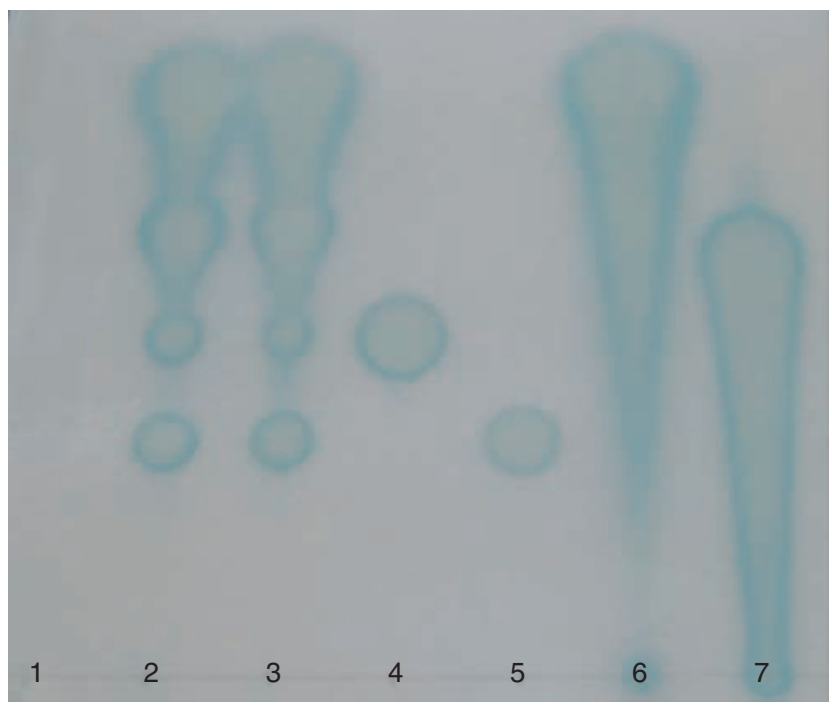

Figure 1 Chromatograph of extracts from Pantoea CWB304 and Pantoea CWB304 panI::Tn5. Samples extracted from culture supernatants of the wild-type, panI mutant and AHL standards were chromatographed on $\mathrm{C}_{18}$ reversed-phase TLC plates and visualized with the $A$. tumefaciens reporter strain NTL4 pZLR5. Samples are for the following extracts from bacterial strains and AHL standards: 1, Pantoea CWB304 panI::Tn5 (AHL-deficient); 2, Pantoea CWB304 extract A; 3, Pantoea CWB304 extract B; 4, C7 AHL; 5, C8 AHL; 6, 3-oxo-C6 AHL and 7, 3-oxo-C8 AHL. AHL, $N$-acyl-L-homoserine lactone; CWB, cabbage white butterfly; TLC, thin layer chromatography. 


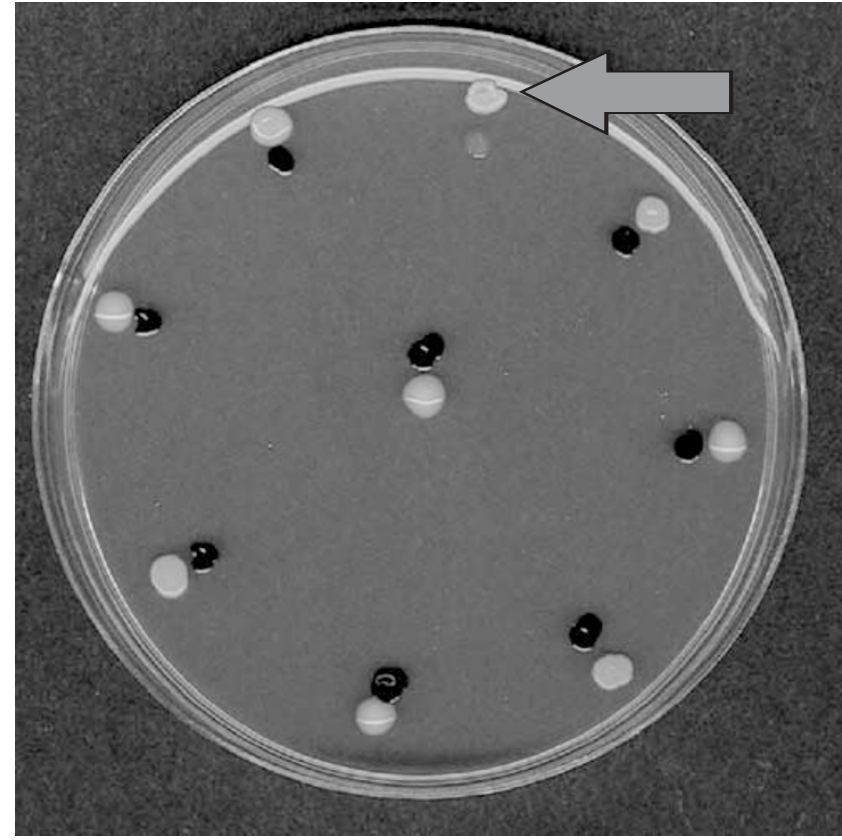

Figure 2 Identification of Pantoea mutants that do not produce AHLs. Each transposon mutant was patched near the C. violaceum CV026 AHL reporter to identify mutants that do not produce AHLs, which do not induce production of violacein (purple pigment) by $C$. violaceum CV026. An AHL-deficient mutant is indicated by the arrow. AHL, $N$-acyl-L-homoserine lactone.

induced in Pantoea CWB304 pSB401 by its own AHLs (Figure 3a), whereas bioluminescence is induced more than 100-fold in Pantoea CWB304 panI::Tn5 pSB401 when exogenous AHLs are provided (Figures 3a and b).

We characterized the regulation and specificity of QS in Pantoea CWB304 by introducing pPanlux into the wild-type Pantoea and the panI mutant and tested the strains in the absence and presence of exogenous AHLs (Figures 3a and b). The reporter plasmid, pPanlux, carries a fusion of the panI promoter region and the promoterless luxCDABE reporter genes. The expression of panI in Pantoea CWB304 panI::Tn5 pPanlux was induced nearly 80fold in late logarithmic growth phase (Figure 4), which suggests that panI is regulated in a densitydependent manner indicative of positive feedback regulation of the AHL synthase.

To determine the nature and specificity of QS regulation in Pantoea CWB304, we introduced the pPanlux construct into the AHL-deficient mutant generating Pantoea CWB304 panI::Tn5 pPanlux. Bioluminescence was induced by the exogenous addition of AHLs, further supporting the conclusion that panI is regulated in a cell density-dependent manner by AHLs (Figure 3b).

To assess the activities of AHLs that induce QS in Pantoea CWB304, we induced Pantoea CWB304 panI::Tn5 pPanlux with various concentrations of C6 AHL, C8 AHL, 3-oxo-C6 AHL and 3-oxo-C8 AHL

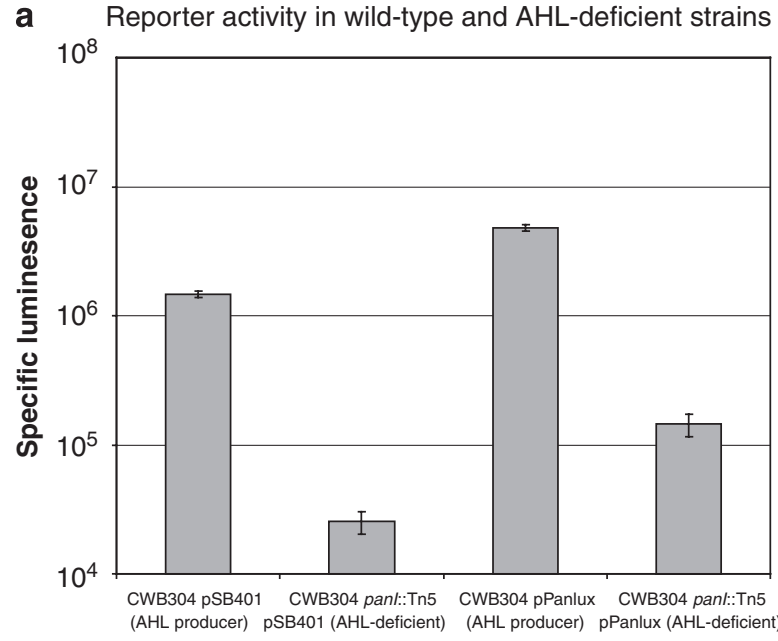

b Reporter activity in wild-type and AHL-deficient strains

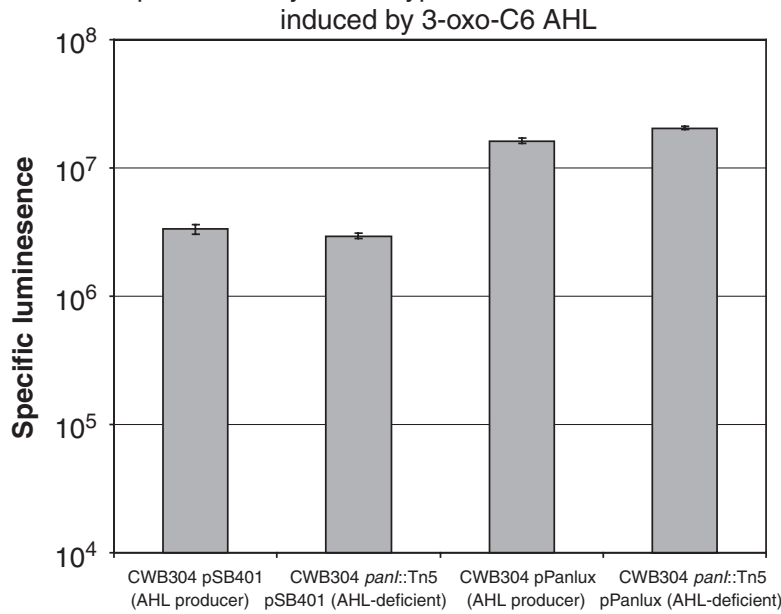

Figure 3 QS activity in Pantoea reporter strains. Pantoea strains were grown in LB media and specific luminescence induced by endogenous AHL production and background reporter activity (a) and the addition of $50 \mathrm{~nm}$ synthetic 3-oxo-C6 AHL (b) were measured. Values represent means of specific luminescence, which is relative luminescence per unit of absorbance $\left(\mathrm{OD}_{600}\right)$, of four replicates after $8 \mathrm{~h}$ of growth. Error bars indicate the s.d. AHL, $N$-acyl-L-homoserine lactone; QS, quorum sensing.

(Figure 5). Synthetic 3-oxo-C6 AHL and 3-oxo-C8 AHL induced luminescence greater than 20-fold at concentrations as low as 5 and $250 \mathrm{nM}$, respectively after $4 \mathrm{~h}$ of growth. C6 and C8 AHLs failed to induce strong luminescence at concentrations up to $500 \mathrm{nM}$. These findings provide evidence that panI is positively regulated in a concentration-dependent manner and that 3-oxo-C6 AHL is likely the preferred ligand for the regulation of the PanIR QS system.

\section{Cell-cell communication in the CWB larval midgut} To determine whether bacteria produce and respond to AHL signal molecules in insecta, bioluminescent reporter strains in various combinations were administered orally to CWB larvae reared under 


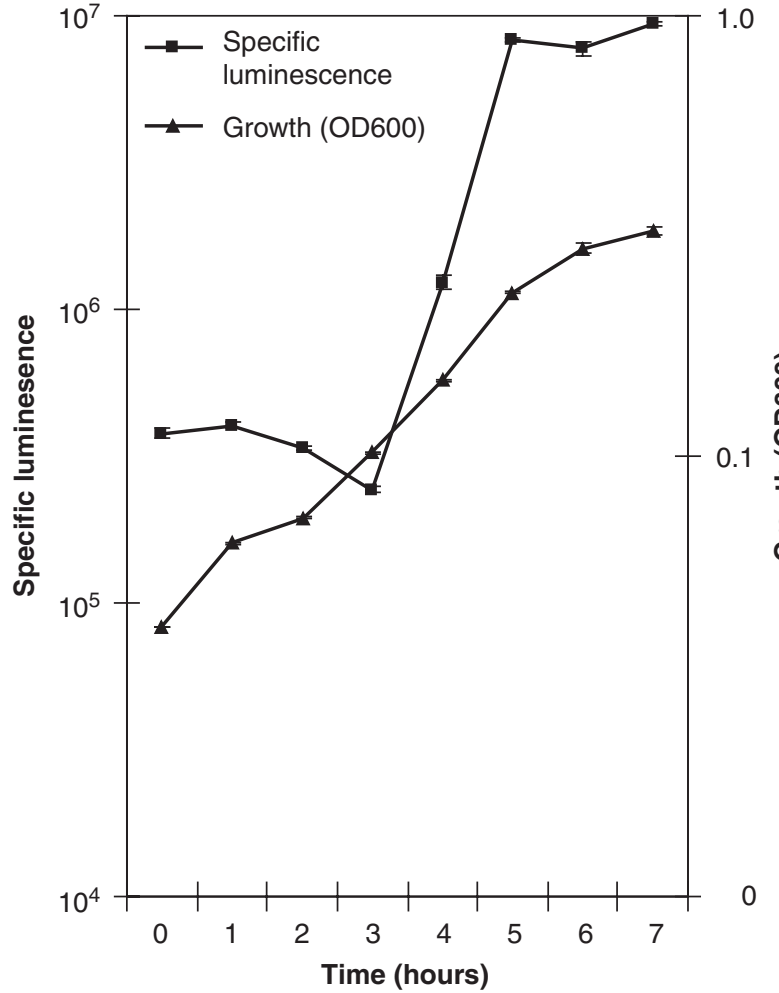

Figure 4 Cell density-dependent regulation of panI. CWB304 pPanlux, which contains a panI-luxCDABE transcriptional fusion, was grown in LB medium, and specific luminescence ( $\square$ ) and optical density ( $\boldsymbol{\Delta}$ ) were measured hourly. The specific luminescence of the bacterial cultures was quantified as relative light units (RLU) per unit of absorbance $\left(\mathrm{OD}_{600}\right)$. Mean values and s.d. are shown for three replicates. CWB, cabbage white butterfly; LB medium, Luria-Bertani medium.

sterile conditions and luminescence was detected by an IVIS BLI 100 CCD camera in vivo. The wild type, which contained the AHL biosynthetic gene panI, and the biosensor, pSB401, were luminescent in the gut of the CWB larvae. This result indicates that Pantoea CWB304 pSB401 produces and responds to AHLs in the insect gut (Figure 6).

To determine whether strains exchange and respond to AHLs in the insect gut, the wild-type and the panI mutant harboring pSB401 were established in the gut and bioluminescence detected with a CCD camera (Figure 6). The AHL-negative Pantoea strain responded to AHLs produced by the wild-type strain, suggesting that AHL signals produced by one strain were perceived by the other strain in the alkaline environment of the CWB larval midgut. Bioluminescence was barely detectable in one of the four caterpillars in which the panI mutant harboring pSB401 was established (Figure 6), indicating a low level of background reporter activity or the limited presence of AHL-producing bacteria. Antibiotic resistance profiles of bacteria recovered from the gut indicate that plasmid transfer was not responsible for activation of the reporter. We detected no transconjugants within the limit of detection of this assay, which is 10 bacteria per

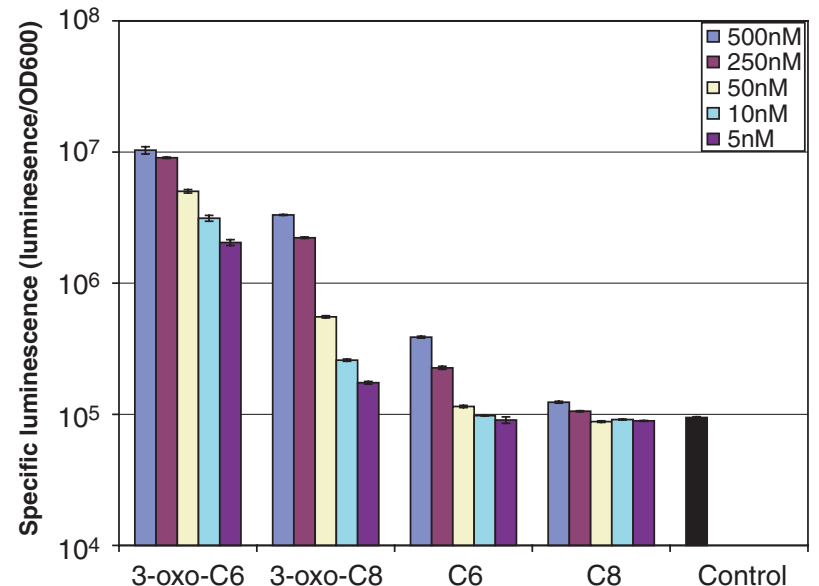

Figure 5 Response of Pantoea CWB304 panI::Tn5 pPanlux to synthetic AHLs. Specific luminescence of CWB304 panI::Tn5 pPanlux was measured in the presence of various synthetic AHLs. Specific luminescence is the luminescence divided by the optical density $\left(\mathrm{OD}_{600}\right)$ of the cultures measured at $4 \mathrm{~h}$. Mean values and s.e. are shown for three replicates. AHL, $N$-acyl-L-homoserine lactone; CWB, cabbage white butterfly.

gut. These results indicate that AHL signal exchange between strains is responsible for activation of the bioluminescent reporter.

Establishment of P. aeruginosa PAO1 as a model pathogen of CWB larvae

To establish $P$. aeruginosa PAO1 as a pathogen of CWB larvae, the colonization and pathogenesis of CWB larvae by $P$. aeruginosa PAO1 were evaluated. PAO1 colonized CWB larvae, establishing a population size of $9 \log$ colony-forming units after 5 days. The population of $P$. aeruginosa PAO1 increased by more than $4 \log$ colony-forming units between days 1 and 3 of the colonization experiment. To establish a causal relationship between $P$. aeruginosa PAO1 and the disease resulting in septicemia and larval death, Koch's postulates were fulfilled. Three independent $P$. aeruginosa isolates cultured from infected CWB larvae were fed to healthy CWB larvae. All of the larvae that were infected with the $P$. aeruginosa isolates developed septicemic symptoms associated with $P$. aeruginosa infection. Isolates of bacteria whose colony morphology resembled that of $P$. aeruginosa were cultured from infected larvae and their identity was verified as $P$. aeruginosa by $16 \mathrm{~S}$ rRNA gene sequence. Control larvae that were not fed $P$. aeruginosa isolates remained healthy, and $P$. aeruginosa was not detected by culturing these larvae.

Role of AHLs in P. aeruginosa virulence in CWB larvae After establishing that $P$. aeruginosa PAO1 is pathogenic to CWB larvae and AHL signals are exchanged in the CWB larvae gut, we explored the question whether AHLs govern bacterial behaviors, 


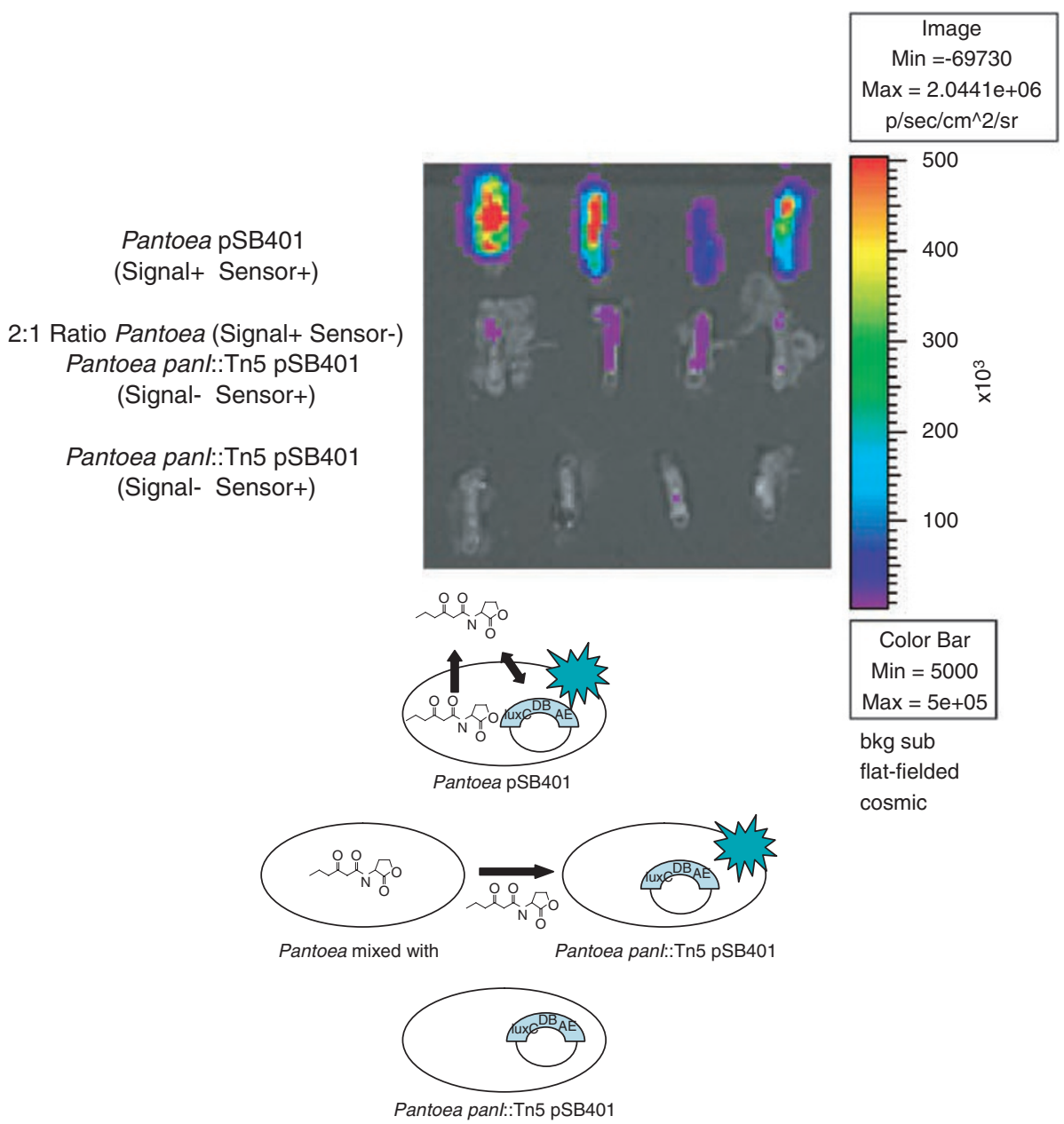

Figure 6 Detection of QS activity and signal exchange in the guts of CWB larvae. Bioluminescence detected in the individual guts of larvae fed Pantoea pSB401 (top row), Pantoea mixed with Pantoea panI::Tn5 pSB401 (middle row) and Pantoea panI::Tn5 pSB401 (bottom row). CWB, cabbage white butterfly; QS, quorum sensing.

specifically pathogenesis. P. aeruginosa PAO1 and $P$. aeruginosa PAO1-JP2, a derivative of PAO1 lacking the genes for AHL synthesis (lasI and rhlI), were fed individually to larvae and mortality was evaluated. The AHL-deficient mutant, PAO1-JP2, induced significantly less mortality $(47 \%)$ than the parent strain $(88 \%)$, indicating that AHL signaling contributes to virulence (Figure 7). Moreover, a synthetic antagonist of LasR, the AHL indole analog $\mathrm{N}$-(indole-3-butanoyl)-L-homoserine lactone (Geske et al., 2005), reduced virulence of the wild type to a level comparable to that of the AHL-deficient mutant (Figure 7).

\section{Discussion}

In this study, we show that QS signals are active in the guts of CWB larvae. Two lines of evidence support this conclusion. First, we demonstrate the exchange of AHL signals in insects in real time with luminescent reporter strains that detect AHLs.
Second, mutations in $P$. aeruginosa that abolish the production of AHLs or treatment of the wild type with a QS inhibitor reduced mortality of larvae compared with larvae infected with wild-type $P$. aeruginosa with no inhibitor. These results are significant because it was previously unknown whether signal exchange occurs in the alkaline environment of the CWB larval midgut. In vitro, AHLs are degraded above $\mathrm{pH} 7$ by lactonolysis, and the open-ring molecule is inactive (Yates et al., 2002). Bacteria residing in the larval guts may alter the $\mathrm{pH}$ of their local microenvironments or exist in biofilms with other organisms that reduce the $\mathrm{pH}$.

Pantoea CWB304 isolated from the gut of CWB larvae produces AHLs that regulate expression of the panI-encoded AHL synthase, which is regulated by cell density. Pantoea CWB304 is most sensitive to 3-oxo-C6 AHL. It is unknown whether panI from Pantoea CWB304 produces all four of the AHLs detected in broth culture in the insect gut or whether there are additional AHL synthases in the genome of Pantoea CWB304 that are regulated by the products 


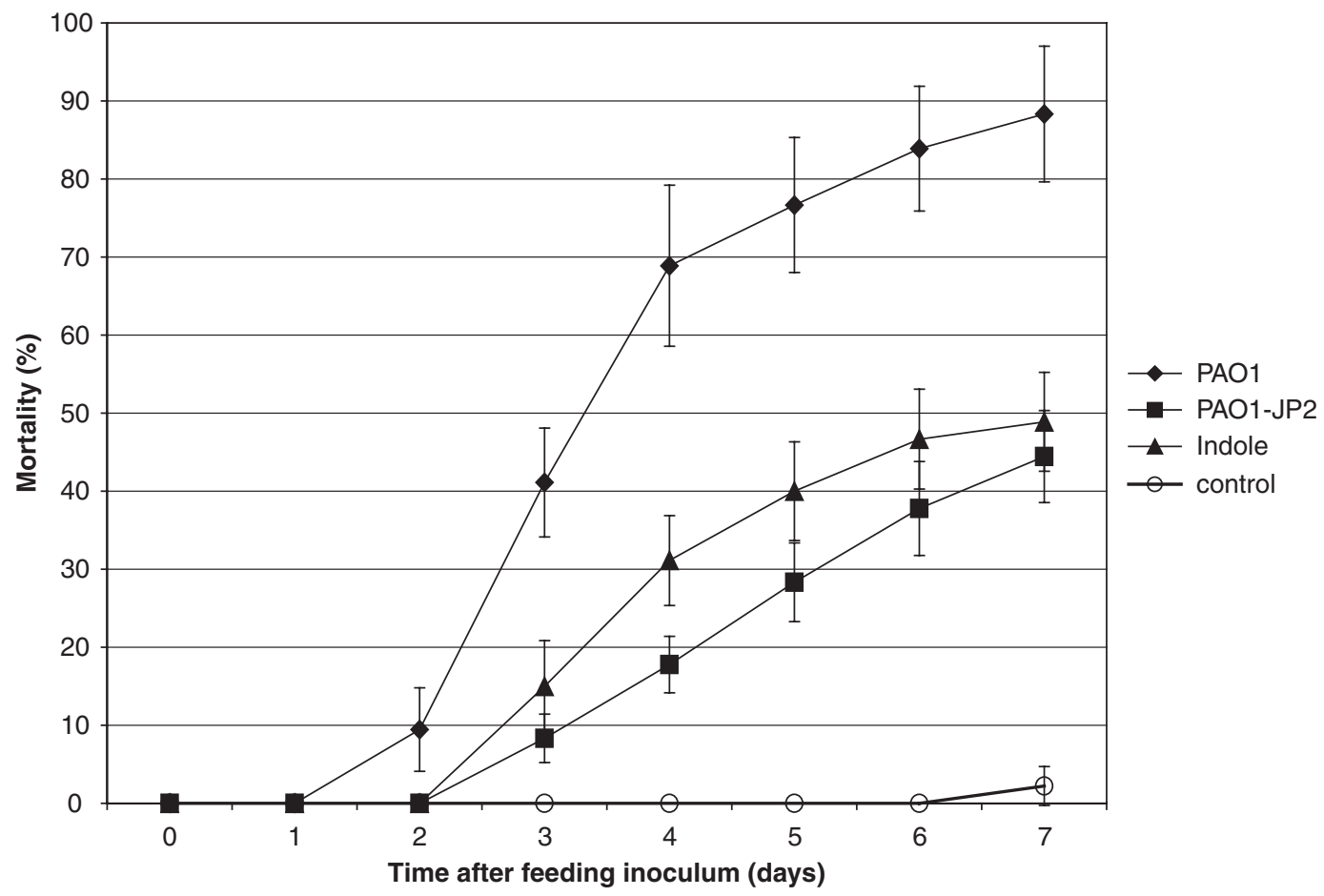

Figure 7 Mortality of cabbage white butterfly larvae fed $P$. aeruginosa strains and the QS analog indole inhibitor. Treatments include

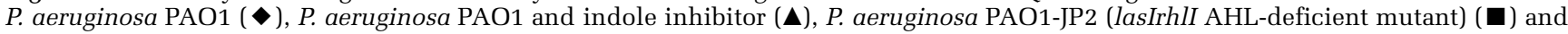
no $P$. aeruginosa PAO1 control $(O)$. Values represent the mean mortality as a percentage of 18 larvae per treatment replicated in five independent experiments. Error bars are the s.e. AHL, $N$-acyl-L-homoserine lactone; QS, quorum sensing.

of panI. The production of other AHLs may be important for crosstalk or regulation of unknown LuxR-type receptors in Pantoea.

Our data indicate that QS signals contribute to pathogenesis of $P$. aeruginosa PAO1 in CWB larvae as in other host-pathogen systems (Tan et al., 1999; Pearson et al., 2000). However, the QS inhibitor and a $P$. aeruginosa mutant deficient in AHL signal production did not completely abolish pathogenicity, indicating that pathogenicity in $P$. aeruginosa is not controlled solely by the production and sensing of AHLs. It is possible that additional signaling systems are involved. The structurally dissimilar $P$. aeruginosa signal, 2-heptyl-3-hydroxy-4-quinolone, plays a significant role in the transcription of $P$. aeruginosa virulence genes (McGrath et al., 2004) and may play a partial role in $P$. aeruginosa pathogenicity of CWB larvae.

The majority of microorganisms exist in multispecies communities in which complex signaling networks are believed to regulate the behavior of the community. Studies of model systems have provided the foundation and principles underlying many microbial interactions, but deciphering the networks that govern the entire community is challenging. To address this challenge, we have developed the multispecies community of the CWB larval gut to study the largely unexplored role of signaling in communities.

Future work will focus on characterizing the Pantoea CWB304 genes that are regulated by QS in the guts of CWB larvae. We will investigate the use of other AHL analog inhibitors on QS inhibition in vivo by monitoring the activity of QS reporter strains in real time. The work reported here demonstrates the use of the CWB larval gut and its associated microbial community as a model system to study signaling interactions in communities. This model invertebrate system may also be used to study additional mechanisms of virulence regulation and the efficacy and non-target effects of possible therapeutics that modulate AHL-dependent QS systems.

\section{Acknowledgements}

We are grateful to Ronald Binder for generously providing synthetic AHLs; to Professors Leo Eberl, Michael Givskov, Max Teplitski and Barbara Iglewski for providing plasmids and strains; and to Gary Splitter and Gireesh Rajashekara for the assistance with and use of the IVIS BLI 100 CCD camera. We are also grateful to Professor Kenneth Raffa for guidance and use of his insect-rearing facilities and Anders Boyd for sharing data on the midgut $\mathrm{pH}$. BRB was supported by an Advanced Opportunity Fellowship from the University of Wisconsin-Madison Graduate School. This work was supported by the Howard Hughes Medical Institute, NIH Grant no. 5RO1GM75830-2 and the University of Wisconsin-Madison College of Agricultural and Life Sciences Hatch Project no. 4038. Research in the Blackwell lab is supported in part by the NSF (CHE0449959), Burroughs Welcome Foundation, Johnson \& 
Johnson and Greater Milwaukee Foundation Shaw Scientist Program. HEB is an Alfred P Sloan Foundation Fellow and a Research Corporation Cottrell Scholar. GDG was supported by an American Chemical Society Division of Medicinal Chemistry predoctoral fellowship. CJR was supported by the Biotechnology Training Program (NIH 5 T32 GM08349) and an Advanced Opportunity Fellowship from the University of Wisconsin-Madison Graduate School.

\section{References}

Altschul SF, Madden TL, Schaffer AA, Zhang J, Zhang Z, Miller W et al. (1997). Gapped BLAST and PSI-BLAST: a new generation of protein database search programs. Nucleic Acids Res 25: 3389-3402.

Andersen JB, Heydorn A, Hentzer M, Eberl L, Geisenberger O, Christensen BB et al. (2001). gfp-based $N$-acyl homoserine-lactone sensor systems for detection of bacterial communication. Appl Environ Microbiol 67: $575-585$.

Berenbaum M (1980). Adaptive significance of midgut $\mathrm{pH}$ in larval Lepidoptera. Am Nat 115: 138-146.

Broderick NA, Raffa KF, Goodman RM, Handelsman J. (2004). Census of the bacterial community of the gypsy moth larval midgut by using culturing and culture-independent methods. Appl Environ Microbiol 70: 293-300.

Carlier A, Uroz S, Smadja B, Fray R, Latour X, Dessaux Y et al. (2003). The $\mathrm{Ti}$ plasmid of Agrobacterium tumefaciens harbors an attM-paralogous gene, aiiB, also encoding $N$-acyl homoserine lactonase activity. Appl Environ Microbiol 69: 4989-4993.

Cui Y, Chatterjee A, Liu Y, Dumenyo CK, Chatterjee AK. (1995). Identification of a global repressor gene, $\operatorname{rsm} A$, of Erwinia carotovora subsp. carotovora that controls extracellular enzymes, $\mathrm{N}$-(3-oxohexanoyl)-L-homoserine lactone, and pathogenicity in soft-rotting Erwinia spp. J Bacteriol 177: 5108-5115.

Dong YH, Gusti AR, Zhang Q, Xu JL, Zhang LH. (2002). Identification of quorum-quenching $N$-acyl homoserine lactonases from Bacillus species. Appl Environ Microbiol 68: 1754-1759.

Engebrecht J, Silverman M. (1984). Identification of genes and gene products necessary for bacterial bioluminescence. Proc Natl Acad Sci USA 81: 4154-4158.

Fuqua WC, Winans SC, Greenberg EP. (1994). Quorum sensing in bacteria: the LuxR-LuxI family of cell density-responsive transcriptional regulators. J Bacteriol 176: 269-275.

Geske GD, Wezeman RJ, Siegel AP, Blackwell HE. (2005). Small molecule inhibitors of bacterial quorum sensing and biofilm formation. J Am Chem Soc 127: 12762-12763.

Givskov M, de Nys R, Manefield M, Gram L, Maximilien R, Eberl L et al. (1996). Eukaryotic interference with homoserine lactone-mediated prokaryotic signalling. J Bacteriol 178: 6618-6622.

Guan C, Ju J, Borlee BR, Williamson LL, Shen B, Raffa KF et al. (2007). Signal mimics derived from a metagenomic analysis of the gypsy moth gut microbiota. Appl Environ Microbiol 73: 3669-3676.

Latifi A, Winson MK, Foglino M, Bycroft BW, Stewart GS, Lazdunski A et al. (1995). Multiple homologues of LuxR and LuxI control expression of virulence determinants and secondary metabolites through quorum sensing in Pseudomonas aeruginosa PAO1. Mol Microbiol 17: 333-343.

Leadbetter JR, Greenberg EP. (2000). Metabolism of acyl-homoserine lactone quorum-sensing signals by Variovorax paradoxus. J Bacteriol 182: 6921-6926.

Lequette Y, Lee JH, Ledgham F, Lazdunski A, Greenberg EP. (2006). A distinct QscR regulon in the Pseudomonas aeruginosa quorum-sensing circuit. J Bacteriol 188: $3365-3370$.

Lesprit P, Faurisson F, Join-Lambert O, Roudot-Thoraval F, Foglino M, Vissuzaine C et al. (2003). Role of the quorum-sensing system in experimental pneumonia due to Pseudomonas aeruginosa in rats. Am J Respir Crit Care Med 167: 1478-1482.

Lin YH, Xu JL, Hu J, Wang LH, Ong SL, Leadbetter JR et al. (2003). Acyl-homoserine lactone acylase from Ralstonia strain XJ12B represents a novel and potent class of quorum-quenching enzymes. Mol Microbiol 47: 849-860.

Lithgow JK, Wilkinson A, Hardman A, Rodelas B, Wisniewski-Dye F, Williams $\mathrm{P}$ et al. (2000). The regulatory locus cinRI in Rhizobium leguminosarum controls a network of quorum-sensing loci. Mol Microbiol 37: 81-97.

Manefield M, Turner SL. (2002). Quorum sensing in context: out of molecular biology and into microbial ecology. Microbiology 148: 3762-3764.

Martinelli D, Grossmann G, Sequin U, Brandl H, Bachofen R. (2004). Effects of natural and chemically synthesized furanones on quorum sensing in Chromobacterium violaceum. BMC Microbiol 4: 25.

McClean KH, Winson MK, Fish L, Taylor A, Chhabra SR, Camara $\mathrm{M}$ et al. (1997). Quorum sensing and Chromobacterium violaceum: exploitation of violacein production and inhibition for the detection of $N$-acylhomoserine lactones. Microbiology 143: 3703-3711.

McGrath S, Wade DS, Pesci EC. (2004). Dueling quorumsensing systems in Pseudomonas aeruginosa control the production of the Pseudomonas quinolone signal (PQS). FEMS Microbiol Lett 230: 27-34.

Michael B, Smith JN, Swift S, Heffron F, Ahmer BM. (2001). SdiA of Salmonella enterica is a LuxR homolog that detects mixed microbial communities. J Bacteriol 183: $5733-5742$.

Miller MB, Bassler BL. (2001). Quorum sensing in bacteria. Annu Rev Microbiol 55: 165-199.

Park SY, Kang HO, Jang HS, Lee JK, Koo BT, Yum DY. (2005). Identification of extracellular $N$-acylhomoserine lactone acylase from a Streptomyces sp. and its application to quorum quenching. Appl Environ Microbiol 71: 2632-2641.

Park SY, Lee SJ, Oh TK, Oh JW, Koo BT, Yum DY et al. (2003). AhlD, an $N$-acylhomoserine lactonase in Arthrobacter sp., and predicted homologues in other bacteria. Microbiology 149: 1541-1550.

Pearson JP, Feldman M, Iglewski BH, Prince A. (2000). Pseudomonas aeruginosa cell-to-cell signaling is required for virulence in a model of acute pulmonary infection. Infect Immun 68: 4331-4334.

Pearson JP, Gray KM, Passador L, Tucker KD, Eberhard A, Iglewski BH et al. (1994). Structure of the autoinducer required for expression of Pseudomonas aeruginosa virulence genes. Proc Natl Acad Sci USA 91: 197-201.

Pearson JP, Passador L, Iglewski BH, Greenberg EP. (1995). A second $N$-acylhomoserine lactone signal produced 
by Pseudomonas aeruginosa. Proc Natl Acad Sci USA 92: 1490-1494.

Pearson JP, Pesci EC, Iglewski BH. (1997). Roles of Pseudomonas aeruginosa las and rhl quorum-sensing systems in control of elastase and rhamnolipid biosynthesis genes. J Bacteriol 179: 5756-5767.

Piper KR, Beck von Bodman S, Farrand SK. (1993). Conjugation factor of Agrobacterium tumefaciens regulates $\mathrm{Ti}$ plasmid transfer by autoinduction. Nature 362: $448-450$.

Riedel K, Hentzer M, Geisenberger O, Huber B, Steidle A, $\mathrm{Wu} \mathrm{H}$ et al. (2001). N-acylhomoserine-lactonemediated communication between Pseudomonas aeruginosa and Burkholderia cepacia in mixed biofilms. Microbiology 147: 3249-3262.

Steidle A, Sigl K, Schuhegger R, Ihring A, Schmid M, Gantner S et al. (2001). Visualization of $N$-acylhomoserine lactone-mediated cell-cell communication between bacteria colonizing the tomato rhizosphere. Appl Environ Microbiol 67: 5761-5770.

Tan MW, Rahme LG, Sternberg JA, Tompkins RG, Ausubel FM. (1999). Pseudomonas aeruginosa killing of Caenorhabditis elegans used to identify $P$. aeruginosa virulence factors. Proc Natl Acad Sci USA 96: 2408-2413.

Visick KL, Foster J, Doino J, McFall-Ngai M, Ruby EG. (2000). Vibrio fischeri lux genes play an important role in colonization and development of the host light organ. J Bacteriol 182: 4578-4586.

von Bodman SB, Majerczak DR, Coplin DL. (1998). A negative regulator mediates quorum-sensing control of exopolysaccharide production in Pantoea stewartii subsp. stewartii. Proc Natl Acad Sci USA 95: 7687-7692.

Williamson LL, Borlee BR, Schloss PD, Guan C, Allen HK, Handelsman J. (2005). Intracellular screen to identify metagenomic clones that induce or inhibit a quorumsensing biosensor. Appl Environ Microbiol 71 6335-6344.

Winson MK, Swift S, Fish L, Throup JP, Jorgensen F, Chhabra SR et al. (1998). Construction and analysis of luxCDABE-based plasmid sensors for investigating $N$-acyl homoserine lactone-mediated quorum sensing. FEMS Microbiol Lett 163: 185-192.

Wittstock U, Agerbirk N, Stauber EJ, Olsen CE, Hippler M, Mitchell-Olds T et al. (2004). Successful herbivore attack due to metabolic diversion of a plant chemical defense. Proc Natl Acad Sci USA 101: 4859-4864.

Yates EA, Philipp B, Buckley C, Atkinson S, Chhabra SR, Sockett RE et al. (2002). $N$-acylhomoserine lactones undergo lactonolysis in a pH-, temperature-, and acyl chain length-dependent manner during growth of Yersinia pseudotuberculosis and Pseudomonas aeruginosa. Infect Immun 70: 5635-5646. 